\title{
REPRESENTATIONS OF A COMPLEX POINT BY PAIRS OF ORDERED REAL POINTS *
}

\author{
BY \\ W. C. GRAUSTEIN
}

1. Introduction. A complex point $z$ in a complex space of $n$ dimensions, since it depends on $2 n$ real parameters, can be represented by a pair of real points, $X$ and $Y$, of the space. It is reasonable to restrict the choice of $X$ and $Y$ by the following conditions:

$A$. The points $X$ and $Y$ representing $z$ shall be ordered: $X \rightarrow Y$.

$B$. If $z$ is a real point, $X$ and $Y$ shall coincide in $z$.

C. If $z$ is imaginary, $X$ and $Y$ shall be distinct; if $X \rightarrow Y$ represents $z$, $Y \rightarrow X$ shall represent $\bar{z}$, the point conjugate-imaginary to $z$.

$D$. The coördinates of $X$ and $Y$, referred to a rectangular coördinate system, shall be analytic functions, without singularities in the finite domain, of the coördinates of $z$ and $\bar{z}$, referred to this system.

$E$. The representation of $z$ by $X \rightarrow Y$ shall be invariant under a chosen group of real point transformations; that is, the ordered points $X^{\prime} \rightarrow Y^{\prime}$ into which the ordered points $X \rightarrow Y$ are carried by an arbitrary transformation of the group shall represent the point $z^{\prime}$ into which $z$ is carried by the transformation.

If $X \rightarrow Y$ is a representation of $z$ which satisfies these conditions, $Y \rightarrow X$ is also a representation of $z$ satisfying them. The two representations shall be called inverse.

There are two pairs of inverse representations satisfying the prescribed conditions which are well known: those of Laguerre in the plane, whereby $z$ is represented by the real points of intersection, taken in the one or the other order, of the minimal lines through $z$ and $\bar{z}$; and those of Marie in the plane and in space, in which the real points of the representation lie on the line $z \bar{z}$, have the same midpoint as $z$ and $\bar{z}$, and are at a distance apart whose square is the negative of the square of the distance $z \bar{z}$. $\dagger$ The two Marie representations are invariant under the group of affine transformations, whereas those of Laguerre are unchanged by the direct circular transformations of the plane.

This paper deals with the determination of all the representations $X \rightarrow Y$

\footnotetext{
* Presented to the Society, December 31, 1919.

$\dagger$ For a review of the literature concerning these representations, see E. Study, Vorlesungen über ausgewählte Gegenstände der Geometrie, erstes Heft; Ebene analytische Kurven und zu ihnen gehörige Abbildungen, Leipzig, 1911; J. L. Coolidge, Geometry in the Complex Domain (in press). Trans. Am. Math. Soc. 17.
} 
of $z$ which satisfy conditions $A$ to $D$, and are invariant, in compliance with $E$, under a given group. In $\S 2$ the problem is formulated analytically for an arbitrary group. In the subsequent sections, 3-7, it is solved for various subgroups of the projective group, for the projective group itself, and for the group of direct circular transformations of the plane. Only in the case of the projective group are no representations obtained.

Given a group, $G$, of direct transformations and the set, $S$, of corresponding indirect transformations, it is of importance to determine, among the representations invariant under $G$, those which are invariant also under $S$, and secondly, those which are inverted, i.e., carried into their inverses, by $S$. It turns out that all the representations invariant under $G$ are invariant also under $S$, except when $G$ is the group of direct circular transformations, or when $G$ is either the group of motions or of direct transformations of similarity in the plane.* In the latter cases certain representations of the totality invariant under $G$ are unchanged by $S$, whereas certain others are inverted by $S(\S 8)$. Moreover, it is only in these same cases that the representations are of exceptional nature in comparison with those obtained for the same group applied in a space of different dimensionality.

2. Analytic formulation of the problem. Let $\left(z_{1}, z_{2}, \cdots, z_{n}\right)$, where

$$
z_{i}=x_{i}+i y_{i} \quad(i=1,2, \cdots, n),
$$

be the coördinates, referred to a rectangular cartesian system of axes, of the point $z$, and let the coördinates of $X$ and $Y$, referred to the same system, be respectively $\left(X_{1}, X_{2}, \cdots, X_{n}\right)$ and $\left(Y_{1}, Y_{2}, \cdots, Y_{n}\right)$.

Since the latter sets of coördinates are, by condition $D$, real analytic functions of $z_{1}, z_{2}, \cdots, z_{n}, \bar{z}_{1}, \bar{z}_{2}, \cdots, \bar{z}_{n}$, they are also real analytic functions of the real variables $x_{1}, y_{1}, x_{2}, y_{2}, \cdots, x_{n}, y_{n}$. It is convenient, in light of $B$, to write these functions as follows:

By $C$,

$$
\begin{aligned}
X_{i} & =x_{i}+F_{i}\left(x_{1}, y_{1}, \cdots, x_{n}, y_{n}\right) \\
Y_{i} & =x_{i}+G_{i}\left(x_{1}, y_{1}, \cdots, x_{n}, y_{n}\right) \quad(i=1,2, \cdots, n) .
\end{aligned}
$$

$G_{i}\left(x_{1}, y_{1}, \cdots, x_{n}, y_{n}\right)=F_{i}\left(x_{1},-y_{1}, \cdots, x_{n},-y_{n}\right) \quad(i=1,2, \cdots, n)$.

Conditions $A$ to $D$ are thus covered by demanding that $X_{i}$ and $Y_{i}$ be of the form

$$
\begin{aligned}
& X_{i}=x_{i}+F_{i}\left(x_{1}, y_{1}, \cdots, x_{n}, y_{n}\right), \\
& Y_{i}=x_{i}+F_{i}\left(x_{1},-y_{1}, \cdots, x_{n},-y_{n}\right) \quad(i=1,2, \cdots, n),
\end{aligned}
$$

where $F_{1}, F_{2}, \cdots, F_{n}$ are real functions of the real variables $x_{1}, y_{1}, \cdots, x_{n}, y_{n}$, which are analytic for all finite sets of values, vanish identically when

$$
y_{1}=y_{2}=\cdots=y_{n}=0,
$$

* In this summary of exceptions, one relatively unimportant case has been omitted. 
and are furthermore such that the system of equations

$$
F_{i}\left(x_{1}, y_{1}, \cdots, x_{n}, y_{n}\right)=F_{i}\left(x_{1},-y_{1}, \cdots, x_{n},-y_{n}\right)
$$

has no real solutions in $y_{1}, y_{2}, \cdots, y_{n}$ except the solution $0,0, \cdots, 0$.

Let the equations

$$
z_{i}^{\prime}=T_{i}\left(z_{1}, z_{2}, \cdots, z_{n}\right) \quad(i=1,2, \cdots, n)
$$

represent an arbitrary group of real point transformations. In order that the representation $X \rightarrow Y$ of the point $z$ be invariant under this group, it is necessary and sufficient that

(a) $x_{i}^{\prime}+F_{i}\left(x_{1}^{\prime}, y_{1}^{\prime}, \cdots, x_{n}^{\prime}, y_{n}^{\prime}\right)$

$$
=T_{i}\left(x_{1}+F_{1}\left(x_{1}, y_{1}, \cdots, x_{n}, y_{n}\right), \cdots,\right.
$$

$$
\left.x_{n}+F_{n}\left(x_{1}, y_{1}, \cdots, x_{n}, y_{n}\right)\right) \text {, }
$$

(b) $x_{i}^{\prime}+F_{i}\left(x_{1}^{\prime},-y_{1}^{\prime}, \cdots, x_{n}^{\prime},-y_{n}^{\prime}\right)$

$$
\begin{aligned}
= & T_{i}\left(x_{1}+F_{1}\left(x_{1},-y_{1}, \cdots, x_{n},-y_{n}\right), \cdots,\right. \\
& \left.x_{n}+F_{n}\left(x_{1},-y_{1}, \cdots, x_{n},-y_{n}\right)\right)(i=1,2, \cdots, n) .
\end{aligned}
$$

Thus our problem, finally formulated, consists in solving the system of functional equations (3) for the functions $F_{1}, F_{2}, \cdots, F_{n}$, subject to the conditions stated under (1).

If the transformations (2) are linear, as will frequently be the case,

$$
\begin{aligned}
& x_{i}^{\prime}=T_{i}\left(x_{1}, x_{2}, \cdots, x_{n}\right), \quad y_{i}^{\prime}=T_{i}^{0}\left(y_{1}, y_{2}, \cdots, y_{n}\right) \\
& (i=1,2, \cdots, n),
\end{aligned}
$$

where $T_{i}^{0}$ is $T_{i}$ minus its absolute term. Changing the signs of $y_{1}, y_{2}, \cdots, y_{n}$ then changes the signs of $y_{1}^{\prime}, y_{2}^{\prime}, \cdots, y_{n}^{\prime}$. Consequently, the two systems of equations in (3) are, in this case, each equivalent to the single system

$$
\begin{array}{r}
F_{i}\left(x_{1}^{\prime}, y_{1}^{\prime}, \cdots, x_{n}^{\prime}, y_{n}^{\prime}\right)=T_{i}^{0}\left(F_{1}\left(x_{1}, y_{1}, \cdots, x_{n}, y_{n}\right),\right. \\
\left.\cdots, F_{n}\left(x_{1}, y_{1}, \cdots, x_{n}, y_{n}\right)\right) \quad(i=1,2, \cdots, n) .
\end{array}
$$

3. Representations invariant under the group of translations. Inasmuch as the group of real translations is a subgroup of each of the other groups under which the invariance of representations is to be considered, it is advantageous to deal with it first. Equations (4) then become

$$
\begin{aligned}
F_{i}\left(x_{1}+a_{1}, y_{1}, \cdots, x_{n}+a_{n}, y_{n}\right)=F_{i}\left(x_{1}, y_{1}, \cdots, x_{n}, y_{n}\right) & \\
& (i=1,2, \cdots, n) .
\end{aligned}
$$

Since $a_{1}, a_{2}, \cdots, a_{n}$, the parameters of the translation, are arbitrary, the system is satisfied if and only if $F_{1}, F_{2}, \cdots, F_{n}$ are functions of $y_{1}, y_{2}, \cdots, y_{n}$ alone.

The representations $X \rightarrow Y$ of $z$ invariant under the group of real translations 
are given by

I $\begin{array}{r}X_{i}=x_{i}+F_{i}\left(y_{1}, y_{2}, \cdots, y_{n}\right), \quad Y_{i}=x_{i}+F_{i}\left(-y_{1},-y_{2}, \cdots,-y_{n}\right) \\ (i=1,2, \cdots, n),\end{array}$

where $F_{1}, F_{2}, \cdots, F_{n}$ are arbitrary real analytic functions which vanish for $y_{1}=y_{2}=\cdots=y_{n}=0$ and are such that the system of equations

$$
F_{i}\left(y_{1}, y_{2}, \cdots, y_{n}\right)=F_{i}\left(-y_{1},-y_{2}, \cdots,-y_{n}\right) \quad(i=1,2, \cdots, n)
$$

has no real solutions other than $0,0, \cdots, 0$.

In the case of a group of linear transformations containing the group of translations, (4) can now be replaced by the system of equations

$$
\begin{aligned}
F_{i}\left(y_{1}^{\prime}, y_{2}^{\prime}, \cdots, y_{n}^{\prime}\right)= & T_{i}^{0}\left(F_{1}\left(y_{1}, y_{2}, \cdots, y_{n}\right),\right. \\
& \left.\cdots, F_{n}\left(y_{1}, y_{2}, \cdots, y_{n}\right)\right) \quad(i=1,2, \cdots, n),
\end{aligned}
$$

in which $x_{1}, x_{2}, \cdots, x_{n}, x_{1}^{\prime}, x_{2}^{\prime}, \cdots, x_{n}^{\prime}$ no longer appear.*

4. The group of homothetic transformations. The representation $X \rightarrow Y$ is invariant under the group of real homothetic transformations

$$
z_{i}^{\prime}=\rho z_{i}+a_{i}, \quad \rho \neq 0 \quad(i=1,2, \cdots, n),
$$

if and only if the system (5), formed for this group, namely

$$
F_{i}\left(\rho y_{1}, \rho y_{2}, \cdots, \rho y_{n}\right)=\rho F_{i}\left(y_{1}, y_{2}, \cdots, y_{n}\right) \quad(i=1,2, \cdots, n),
$$

is satisfied. But then, regardless of whether $\rho$ is unrestricted or takes on only positive values, $F_{1}, F_{2}, \cdots, F_{n}$ must be linear and homogeneous in $y_{1}, y_{2}, \cdots, y_{n}$.

The representations $X \rightarrow Y$ of $z$ invariant under the group of either direct or general homothetic transformations are

II

$$
X_{i}=x_{i}+\sum_{j=1}^{n} k_{i j} y_{j}, \quad Y_{i}=x_{i}-\sum_{j=1}^{n} k_{i j} y_{j} \quad(i=1,2, \cdots, n),
$$

where the $k_{i j}$ are real constants subject only to the restriction that the $n$-rowed determinant $\left|k_{i j}\right|$ does not vanish.

* The equations (5) are also necessary and sufficient conditions that the real analytic point transformation

$$
y_{i}^{\prime}=F_{i}\left(y_{1}, y_{2}, \cdots, y_{n}\right)
$$$$
(i=1,2, \cdots, n)
$$

be commutative with each transformation of the group of real linear transformations

$$
y_{i}^{\prime}=T_{i}^{0}\left(y_{1}, y_{2}, \cdots, y_{n}\right) \quad(i=1,2, \cdots, n)
$$

leaving the origin invariant. Accordingly, as we solve (5) in the subsequent paragraphs for various groups $(b)$, we determine at the same time, for each particular group $(b)$, the group of transformations $(a)$, each of which is commutative with every transformation $(b)$. Thus we find, for example, in $\$ 6$, that the group of transformations commutative with the group of motions about the origin in the plane is

$$
y_{1}^{\prime}=y_{1} R_{1}\left(r^{2}\right)+y_{2} R_{2}\left(r^{2}\right), \quad y_{2}^{\prime}=-y_{1} R_{2}\left(r^{2}\right)+y_{2} R_{1}\left(r^{2}\right),
$$

where $r^{2}=y_{1}^{2}+y_{2}^{2}$ and $R_{1}, R_{2}$ are analytic functions of $r^{2}$, not both zero. 
The condition $\left|k_{i j}\right| \neq 0$ is necessary and sufficient, not merely that $X$ and $Y$ coincide only when $z$ is real, but also that the $2 n$ equations II can be solved uniquely for $x_{1}, x_{2}, \cdots, x_{n}, y_{1}, y_{2}, \cdots, y_{n}$ and hence the coördinates of $z$ found in terms of those of $X$ and $Y$. In other words, the correspondence between the ordered point pairs $X \rightarrow Y$ and the points $z$ under a representation II is one-to-one without exception in the finite domain.

5. The affine and projective groups. In order that the representation II be invariant under the $n$ transformations

$$
z_{q}^{\prime}=-z_{q}, \quad z_{i}^{\prime}=z_{i} \quad(q=1,2, \cdots, n),
$$

where in each transformation $i$ takes on all the integral values from 1 to $n$ except the fixed value $q$, it is necessary that $k_{i j}=0, i \neq j$. If the resulting representation is to be unchanged by each of the transformations

$$
z_{q}^{\prime}=z_{r}, \quad z_{r}^{\prime}=z_{q}, \quad z_{i}^{\prime}=z_{i} \quad(q \neq r),
$$

where in each transformation $q$ and $r$ are fixed and $i$ takes on the remaining values from 1 to $n$, it follows that $k_{11}=k_{22}=\cdots=k_{n n}$. A necessary condition, therefore, that II be invariant under the affine group is that $k_{i j}=0$, $i \neq j$, and $k_{i i}=k$ for all $i$. That this condition is also sufficient can be easily verified.

If, instead of all real affine transformations, merely those for which the determinant of the coefficients is positive had been admitted, the result would have been the same.

The representations $X \rightarrow Y$ of $z$ invariant under the group of either direct or general affine transformations are

III

$$
X_{i}=x_{i}+k y_{i}, \quad Y_{i}=x_{i}-k y_{i} \quad(i=1,2, \cdots, n),
$$

where $k$ is an arbitrary constant, $\neq 0$.

The two inverse representations for which $k= \pm 1$ are the representations of Marie.

If III is to be invariant under the projective group, it must be unchanged by the transformation

$$
z_{1}^{\prime}=\frac{1}{z_{1}}, \quad z_{i}^{\prime}=\frac{z_{i}}{z_{1}} \quad(i=2, \cdots, n) .
$$

But then the first of the equations (3) necessitates that $k^{2}=-1$.

There is no representation $X \rightarrow Y$ of $z$ which is invariant under the projective group.

6. The group of motions. The treatment in this case varies with the dimensionality of the space.

A. $n=1$. The group of motions is the group of translations. According to I, the invariant representations are

IVA

$$
X_{1}=x_{1}+y_{1} \Phi\left(y_{1}\right), \quad Y_{1}=x_{1}-y_{1} \Phi\left(y_{1}\right),
$$


where $\Phi\left(y_{1}\right)$ is a real analytic function and the equation $\Phi\left(y_{1}\right)=-\Phi\left(-y_{1}\right)$ is satisfied by no real value of $y_{1}$ except perhaps $y_{1}=0$.

$B$. $n=2$. The representation must be of the form

$$
X_{i}=x_{i}+F_{i}\left(y_{1}, y_{2}\right), \quad Y_{i}=x_{i}+F_{i}\left(-y_{1},-y_{2}\right) \quad(i=1,2),
$$

and be invariant under the transformations

$$
z_{1}^{\prime}=\cos \phi z_{1}-\sin \phi z_{2}+a_{1}, \quad z_{2}^{\prime}=\sin \phi z_{1}+\cos \phi z_{2}+a_{2} .
$$

That is, $F_{1}\left(y_{1}, y_{2}\right), F_{2}\left(y_{1}, y_{2}\right)$ must be solutions of the system of equations, obtained from (5),

where

$$
\begin{aligned}
& F_{1}\left(y_{1}^{\prime}, y_{2}^{\prime}\right)=\cos \phi F_{1}\left(y_{1}, y_{2}\right)-\sin \phi F_{2}\left(y_{1}, y_{2}\right), \\
& F_{2}\left(y_{1}^{\prime}, y_{2}^{\prime}\right)=\sin \phi F_{1}\left(y_{1}, y_{2}\right)+\cos \phi F_{2}\left(y_{1}, y_{2}\right),
\end{aligned}
$$

$$
y_{1}^{\prime}=\cos \phi y_{1}-\sin \phi y_{2}, \quad y_{2}^{\prime}=\sin \phi y_{1}+\cos \phi y_{2} \text {. }
$$

For $\phi=\pi$, equations ( 7 ) become the conditions that $F_{1}, F_{2}$ be odd functions of $y_{1}, y_{2}$. Consequently, if $F_{i}$ is written in the form

$$
\begin{array}{r}
F_{i}\left(y_{1}, y_{2}\right)=\frac{F_{i}\left(y_{1}, y_{2}\right)+F_{i}\left(y_{1},-y_{2}\right)}{2}+\frac{F_{i}\left(y_{1}, y_{2}\right)+F_{i}\left(-y_{1}, y_{2}\right)}{2} \quad(i=1,2),
\end{array}
$$

the first quotient on the right is even in $y_{2}$, odd in $y_{1}$ and hence identically zero for $y_{1}=0$, whereas the second is even in $y_{1}$, odd in $y_{2}$ and so identically zero for $y_{2}=0$. Accordingly,

$$
F_{i}\left(y_{1}, y_{2}\right)=y_{1} \boldsymbol{H}_{i}^{\prime \prime}\left(y_{1}, y_{2}\right)+y_{2} F_{i}^{\prime \prime}\left(y_{1}, y_{2}\right) \quad(i=1,2) \text {, }
$$

where each of the functions $F_{1}^{\prime}, F_{1}^{\prime \prime}, F_{2}^{\prime}, F_{2}^{\prime \prime}$ is analytic and is even in $y_{1}$, in $y_{2}$, and hence in $y_{1}, y_{2}$.

In the equations obtained from (7) by substituting for $F_{1}$ and $F_{2}$ their values as given by (8), replace $\phi$ by $-\phi, y_{1}$ by $-y_{1}$, and combine by addition and subtraction each of the resulting equations with that from which it arose. The system obtained is

$$
\begin{aligned}
& y_{1}^{\prime} F_{1}^{\prime}\left(y_{1}^{\prime}, y_{2}^{\prime}\right)=y_{1} \cos \phi F_{1}^{\prime}\left(y_{1}, y_{2}\right)-y_{2} \sin \phi F_{2}^{\prime \prime}\left(y_{1}, y_{2}\right), \\
& y_{2}^{\prime} F_{2}^{\prime \prime}\left(y_{1}^{\prime}, y_{2}^{\prime}\right)=y_{1} \sin \phi F_{1}^{\prime}\left(y_{1}, y_{2}\right)+y_{2} \cos \phi F_{2}^{\prime \prime}\left(y_{1}, y_{2}\right), \\
& y_{1}^{\prime} F_{2}^{\prime}\left(y_{1}^{\prime}, y_{2}^{\prime}\right)=y_{1} \cos \phi F_{2}^{\prime}\left(y_{1}, y_{2}\right)+y_{2} \sin \phi F_{1}^{\prime \prime}\left(y_{1}, y_{2}\right), \\
& y_{2}^{\prime} F_{1}^{\prime \prime}\left(y_{1}^{\prime}, y_{2}^{\prime}\right)=-y_{1} \sin \phi F_{2}^{\prime}\left(y_{1}, y_{2}\right)+y_{2} \cos \phi F_{1}^{\prime \prime}\left(y_{1}, y_{2}\right) .
\end{aligned}
$$

These equations reduce, for $\phi=\pi / 2$, to

$$
F_{2}^{\prime}\left(y_{1}, y_{2}\right)=-F_{1}^{\prime \prime}\left(y_{2}, y_{1}\right), \quad F_{2}^{\prime \prime}\left(y_{1}, y_{2}\right)=F_{1}^{\prime}\left(y_{2}, y_{1}\right) \text {. }
$$

When the values for $F_{2}^{\prime}$ and $F_{2}^{\prime \prime}$ given by (10) are substituted in (9) and $y_{2}$ is 
set equal to zero, the four resulting equations yield immediately the identities

$$
\begin{aligned}
& F_{1}^{\prime}\left(y_{1} \cos \phi, y_{1} \sin \phi\right)=F_{1}^{\prime}\left(y_{1} \sin \phi, y_{1} \cos \phi\right), \\
& F_{1}^{\prime \prime}\left(y_{1} \cos \phi, y_{1} \sin \phi\right)=F_{1}^{\prime \prime}\left(y_{1} \sin \phi, y_{1} \cos \phi\right),
\end{aligned}
$$

in the independent variables $y_{1}$ and $\phi$. Consequently, $F_{1}^{\prime}\left(y_{1}, y_{2}\right)$ and $F_{1}^{\prime \prime}\left(y_{1}, y_{2}\right)$ are symmetric functions of $y_{1}, y_{2}$, and (10) becomes

$$
F_{2}^{\prime}\left(y_{1}, y_{2}\right)=-F_{1}^{\prime \prime}\left(y_{1}, y_{2}\right), \quad F_{2}^{\prime \prime}\left(y_{1}, y_{2}\right)=F_{1}^{\prime}\left(y_{1}, y_{2}\right) \text {. }
$$

But the system (9) then reduces to

$$
F_{1}^{\prime}\left(y_{1}^{\prime}, y_{2}^{\prime}\right)=F_{1}^{\prime}\left(y_{1}, y_{2}\right), \quad F_{1}^{\prime \prime}\left(y_{1}^{\prime}, y_{2}^{\prime}\right)=F_{1}^{\prime \prime}\left(y_{1}, y_{2}\right) \text {. }
$$

That is, $F_{1}^{\prime}$ and $F_{1}^{\prime \prime}$ are absolute invariants of the group of rotations about the origin, and hence must be of the form

$$
F_{1}^{\prime}=R_{1}\left(r^{2}\right), \quad F_{1}^{\prime \prime}=R_{2}\left(r^{2}\right),
$$

where

$$
r^{2}=y_{1}^{2}+y_{2}^{2} \text {. }
$$

The necessary conditions (8), (11), (12) that $F_{1}\left(y_{1}, y_{2}\right), F_{2}\left(y_{1}, y_{2}\right)$ be solutions of (7) are shown sufficient by direct substitution.

The representations $X \rightarrow Y$ of $z$ invariant under the group of motions in the plane are

IVB

$$
\begin{array}{ll}
X_{1}=x_{1}+y_{1} R_{1}\left(r^{2}\right)+y_{2} R_{2}\left(r^{2}\right), & Y_{1}=x_{1}-y_{1} R_{1}\left(r^{2}\right)-y_{2} R_{2}\left(r^{2}\right), \\
X_{2}=x_{2}-y_{1} R_{2}\left(r^{2}\right)+y_{2} R_{1}\left(r^{2}\right), & Y_{2}=x_{2}+y_{1} R_{2}\left(r^{2}\right)-y_{2} R_{1}\left(r^{2}\right),
\end{array}
$$

where $r^{2}=y_{1}^{2}+y_{2}^{2}$, and $R_{1}, R_{2}$ are real functions of $r^{2}$, analytic for all finite values and not vanishing simultaneously except perhaps when $r^{2}=0$.

C. $n \geqq 3$. In case $n=3$, the functions $F_{1}, F_{2}, F_{3}$ in the representation

$$
X_{i}=x_{i}+F_{i}\left(y_{1}, y_{2}, y_{3}\right), \quad Y_{i}=x_{i}+F_{i}\left(-y_{1},-y_{2},-y_{3}\right)
$$

$$
(i=1,2,3)
$$

are to be determined from the system of equations

$$
F_{i}\left(y_{1}^{\prime}, y_{2}^{\prime}, y_{3}^{\prime}\right)=\sum_{j=1}^{3} a_{i j} F_{j}\left(y_{1}, y_{2}, y_{3}\right) \quad(i=1,2,3)
$$

where

$$
y_{i}^{\prime}=\sum_{j=1}^{3} a_{i j} y_{j} \quad(i=1,2,3),
$$

and the determinant $\left|a_{i j}\right|$ is orthogonal and has the value +1 .

In case of an arbitrary rotation about the axis of $z_{3}, y_{3}^{\prime}=y_{3}$ and $y_{1}, y_{2}$ are transformed as for $n=2$. The last equation of (14) reduces to

$$
F_{3}\left(y_{1}^{\prime}, y_{2}^{\prime}, y_{3}\right)=F_{3}\left(y_{1}, y_{2}, y_{3}\right) \text {, }
$$

and the first two to the equations (7), with the variable $y_{3}$ added in each 
function. Consequently,

$$
\begin{aligned}
& F_{1}=y_{1} F_{3}^{\prime}\left(y_{1}^{2}+y_{2}^{2}, y_{3}\right)+y_{2} F_{3}^{\prime \prime}\left(y_{1}^{2}+y_{2}^{2}, y_{3}\right), \\
& F_{2}=-y_{1} F_{3}^{\prime \prime}\left(y_{1}^{2}+y_{2}^{2}, y_{3}\right)+y_{2} F_{3}^{\prime}\left(y_{1}^{2}+y_{2}^{2}, y_{3}\right), \\
& F_{3}=F_{3}^{\prime \prime \prime}\left(y_{1}^{2}+y_{2}^{2}, y_{3}\right) .
\end{aligned}
$$

Corresponding respectively to the rotations about the axes of $z_{1}$ and $z_{2}$, there are two other sets of equations like (15) and obtainable from (15) by permuting the subscripts $1,2,3$ cyclicly. From the three sets of equations it follows that $F_{1}^{\prime \prime}=F_{2}^{\prime \prime}=F_{3}^{\prime \prime}=0$; for example, since $F_{1}=F_{1}^{\prime \prime \prime}\left(y_{2}^{2}+y_{3}^{2}, y_{1}\right)$, $F_{1}$ is an even function of $y_{2}$, whence, by the first equation of (15), $F_{3}^{\prime \prime}=0$. Therefore

$$
F_{i}=y_{i} F_{j}^{\prime}\left(y_{i}^{2}+y_{k}^{2}, y_{j}\right)=y_{i} F_{k}^{\prime}\left(y_{i}^{2}+y_{j}^{2}, y_{k}\right) \text {, }
$$

where $i, j, k$ are to be permuted cyclicly through the values $1,2,3$. Thus

$$
F_{1}^{\prime}=F_{2}^{\prime}=F_{3}^{\prime}=R\left(r^{2}\right), \quad \text { where } \quad r^{2}=y_{1}^{2}+y_{2}^{2}+y_{3}^{2},
$$

and (13) becomes

$$
X_{i}=x_{i}+y_{i} R\left(r^{2}\right), \quad Y_{i}=x_{i}-y_{i} R\left(r^{2}\right) \quad(i=1,2,3) .
$$

Substitution shows that the functions $F_{i}$ in this representation satisfy (14).

This result for $n=3$ can be generalized by mathematical induction to hold for the case $n>3$.

The representations $X \rightarrow Y$ of $z$ invariant under the group of motions in $n$ dimensional space, where $n \geqq 3$, are

IVC $\quad X_{i}=x_{i}+y_{i} R\left(r^{2}\right), \quad Y_{i}=x_{i}-y_{i} R\left(r^{2}\right) \quad(i=1,2, \cdots, n)$,

where

$$
r^{2}=y_{1}^{2}+y_{2}^{2}+\cdots+y_{n}^{2},
$$

and $R\left(r^{2}\right)$ is a real function analytic for all finite values and not zero except perhaps when $r^{2}=0$.

For the group of motions and reflections the result incorporated in IVC holds for all values of $n$. To establish the truth of this statement for $n \geqq 3$, it is sufficient to show that the representation IVC is invariant under a reflection in one of the coördinate hyperplanes. If $n=2$, the representation must be of the form IVB and be invariant under $z_{1}^{\prime}=z_{1}, z_{2}^{\prime}=-z_{2}$; then $R_{2}\left(r^{2}\right)=0$, and conversely. Finally, IVA is invariant under $z_{1}^{\prime}=-z_{1}$ if and only if $\Phi\left(y_{1}\right)$ is an even function of $y_{1}$, i.e., is of the form $R\left(y_{1}^{2}\right)$.

7. The group of direct transformations of similarity. Necessary and sufficient that the representation $X \rightarrow Y$ be unchanged by the direct transformations of similarity in the cases $n=1, n=2, n \geqq 3$ is the requirement that IVA, IVB, IVC be respectively invariant under an arbitrary stretching from the origin of positive ratio. According to $\S 4$, the arbitrary functions involved in the representations IV are, then, all constants, and conversely. 
The representations $X \rightarrow Y$ of $z$ invariant under the group of direct transformations of similarity are

VA

$$
X_{1}=x_{1}+k y_{1}, \quad Y_{1}=x_{1}-k y_{1},
$$$$
k \neq 0 \text {, if } n=1 \text {, }
$$

VB

$$
X_{1}=x_{1}+k y_{1}+l y_{2}, \quad Y_{1}=x_{1}-k y_{1}-l y_{2},
$$

$$
X_{2}=x_{2}-l y_{1}+k y_{2}, \quad Y_{2}=x_{2}+l y_{1}-k y_{2} \text {, }
$$

$$
k^{2}+l^{2} \neq 0, \text { if } n=2,
$$

VC

$$
X_{i}=x_{i}+k y_{i}
$$

$$
\begin{aligned}
& Y_{i}=x_{i}-k y_{i}, \\
& k \neq 0, \quad i=1,2, \cdots, n, \quad \text { if } \quad n \geqq 3 .
\end{aligned}
$$

Except in the plane, these representations are identical with the representations III invariant under the affine group.

For the group of direct and indirect transformations of similarity the representations are for all $n$ given by VC and are identical with those peculiar to the affine group.

The two inverse representations $\mathrm{VB}$, for which $k=0$ and $l= \pm 1$, namely

VI

$$
\begin{array}{ll}
X_{1}=x_{1} \pm y_{2}, & Y_{1}=x_{1} \mp y_{2}, \\
X_{2}=x_{2} \mp y_{1}, & Y_{2}=x_{2} \pm y_{1},
\end{array}
$$

are the representations of Laguerre. From their geometrical definition $(\S 1)$, it is evident that these representations are invariant under the direct circular transformations. It can be shown that they are the only representations with this property.

8. Representations inverted by a set of transformations. Given a group, $G$, of direct transformations of a certain type and the set, $S$, of indirect transformations of the same type, to determine, among the representations invariant under $G$, those which, though not invariant under $S$, are merely inverted by the transformations of $S$.

It is geometrically evident that a Laguerre representation is inverted by an indirect circular transformation. Of the groups $G$ other than the circular group, every one under which invariant representations exist is linear and contains the group of translations. Hence the representation must be of the form I. In order that it be inverted by a linear transformation (2), it is necessary and sufficient that the functions $F_{i}$ satisfy the system of equations

$$
\begin{array}{r}
F_{i}\left(-y_{1}^{\prime},-y_{2}^{\prime}, \cdots,-y_{n}^{\prime}\right) \\
=T_{i}^{0}\left(F_{1}\left(y_{1}, y_{2}, \cdots, y_{n}\right), \cdots, F_{n}\left(y_{1}, y_{2}, \cdots, y_{n}\right)\right) \\
\quad(i=1,2, \cdots, n),
\end{array}
$$

In $\$ \S 4-7$ it has been shown that, when $G$ is any one of the following groups of direct transformations: homothetic group, affine group, group of motions 
$(n \geqq 3)$, similarity group $(n \neq 2)$, all the representations invariant under $G$ are also invariant under $S$. It is true also that no one of the representations IVA, invariant under the group of motions for $n=1$, is inverted by any transformation of $S$, as can readily be proved by applying (16) with $z_{1}^{\prime}=-z_{1}$ as the transformation used; but in this case, not all the representations are invariant under $S(\S 6)$.

If $G$ is the group of motions (or of direct transformations of similarity) in the plane, the functions $F_{i}$ to be used in (16) are as in IVB (or VB). It is sufficient to choose as the transformation a reflection in a coördinate axis. Then equations (16) reduce to $R_{1}=0$ (or $k=0$ ).

Of the representations IVB invariant under the group of motions in the plane, only those for which $R_{2}=0$ are invariant under reflections, whereas only those for which $R_{1}=0$ are inverted by every reflection.

Of the representations VB invariant under the direct transformations of similarity, only those for which $l=0$ are invariant under the indirect transformations of similarity, whereas only those for which $k=0$ are inverted by every such transformation.

HARVARD UNIVERSITY, Cambridge, Mass. 